The Agriculturists 16(2): 58-64 (2018) ISSN 2304-7321 (Online), ISSN 1729-5211 (Print)

A Scientific Journal of Krishi Foundation

Indexed Journal

DOI: http://dx.doi.org/ 10.3329/agric.v16i02.40343

Impact Factor: 0.568 (GIF, 2015)

\title{
Effects of Plant Growth Regulator on Yield and Economic Benefit of Sweet Pepper (Capsicum annum L.)
}

\author{
S. Akhter, T. Mostarin, K. Khatun, F. Akhter and A. Parvin \\ Department of Horticulture, Sher-e-Bangla Agricultural University, Dhaka, 1207 \\ *Corresponding author and Email: shilaakhter101@gmail.com
}

Received: 21 October $2018 \quad$ Accepted: 25 December 2018

\begin{abstract}
The experiment was conducted in the Horticultural Farm of Sher-e-Bangla Agricultural University, Dhaka, Bangladesh. Plant growth regulators were applied which had significant effect on yield of sweet pepper (Capsicum annum L.). The experiment consisted of two factors. Factor A: Plant growth regulators (four levels) as $\mathrm{G}_{0}$ : Control, $\mathrm{G}_{1}$ : Gibberellic Acid $\left(\mathrm{GA}_{3}\right)$ @ 30 ppm, $\mathrm{G}_{2}$ : 4-Chloro Phenoxy Acetic Acid (4-CPA) @ 45 ppm and $\mathrm{G}_{3}$ : 4-Chloro Phenoxy Acetic Acid (4-CPA) @ 45 ppm + Gibberellic Acid $\left(\mathrm{GA}_{3}\right) @ 30$ ppm and Factor B: Number of spray (three levels) as $\mathrm{N}_{0}$ : Control (no spray), $\mathrm{N}_{1}$ : two spray, $\mathrm{N}_{2}$ : three spray. In case of plant growth regulators, the highest yield (27.77 t/ha) was found from $\mathrm{G}_{3}$ treatment, whereas the lowest $\left(18.87 \mathrm{t} / \mathrm{ha}\right.$ ) was from $\mathrm{G}_{0}$ treatment. For number of spray the maximum yield (26.0 t/ha) was recorded from $\mathrm{N}_{2}$ treatment, while the minimum yield (19.87 $\mathrm{t} / \mathrm{ha}$ ) was from $\mathrm{N}_{0}$ treatment. The results indicated that the highest yield $(31.8 \mathrm{t} / \mathrm{ha})$ was observed from $\mathrm{G}_{3} \mathrm{~N}_{2}$ treatment combination, while the lowest yield (17.5 t/ha) was from $\mathrm{G}_{0} \mathrm{~N}_{0}$ treatment combination. Due to combined effect, the highest yield (31.8 t/ha) with net income (Tk/ha 1416558) and BCR (2.46) was observed from $\mathrm{G}_{3} \mathrm{~N}_{2}$ treatment combination, while the lowest yield $(17.5 \mathrm{t} / \mathrm{ha})$ with net income (Tk/ha 433045) and BCR (1.49) from $\mathrm{G}_{0} \mathrm{~N}_{0}$ treatment combination. Thus, three times spray with (4Chloro Phenoxy Acetic Acid + Gibberellic Acid) may be recommended for achieving the higher growth, yield and economic benefit of sweet pepper.
\end{abstract}

Keywords: Sweet pepper, plant growth regulators, number of spray, yield.

\section{Introduction}

Sweet pepper (Capsicum annum L.) botanically referred to as the genus Capsicumis the member of Solanaceae family. Sweet pepper is relatively non-pungent or less pungent and it is the world second most important vegetables after tomato (AVRDC, 1989). Recent efforts are being made to grow sweet pepper in Bangladesh (Paul, 2009). But the production of sweet pepper is reduced due to flower and fruit drop which is caused by physiological and hormonal imbalance in the plants particularly under unfavourable environments (Erickson and Makhart, 2001). The varying responses of sweet pepper to plant growth regulators have been reported by Changli and Liusheng, (2009). Improvement in pepper growth and yield under $\mathrm{GA}_{3}$ application was observed Vandana and verma, (2014). This might be ascribed to more effective utilization of food for reproductive growth (flowering and fruit set), higher photosynthetic efficiency and enhanced translocation and accumulation of sugars and other metabolites. Another growth regulators 
namely, 4-chlorophenoxy acetic acid has been found to be effective in increasing fruit set and also used in reducing pre- harvest fruit drop and resulting in higher number of fruits and yield.

On the other hand, number of spray play an important role for producing maximum yield. However, information regarding the effectiveness of PGRs and different number of spray on growth and other physiological parameters of commercial pepper cultivars is very little. A detailed and systemic study is needed to find out the optimum concentration and the suitable combination of growth regulators and their number of spray for maximizing the yield of sweet pepper in Bangladesh.

\section{Materials and Methods}

\subsection{Experimental site and experimental frame work}

The experiment was carried out at the horticulture farm, Sher-e-Bangla Agricultural University, Dhaka, Bangladesh during the period from November 2016 to March 2017. The location of the experimental site was at $23.41^{\circ} \mathrm{N}$ latitude and $90.22^{\circ}$ longitude with an elevation of $8.24 \mathrm{~m}$ from sea level. Soil was having the texture of sandy loam with $\mathrm{p}^{\mathrm{H}}$ 5.6. Seeds of sweet pepper variety viz KS 2201 (Krishibid seed) was used as experimental material. The experiment was laid out in Randomized Complete Block Design with three replications. Factor- A had four levels of plant growth regulators viz. $\mathrm{G}_{0-}$ control, $\mathrm{G}_{1}-\mathrm{GA}_{3}$ at $30 \mathrm{ppm}$, $\mathrm{G}_{2}-4-\mathrm{CPA}$ at $45 \mathrm{ppm}, \mathrm{G}_{3}-4-\mathrm{CPA} @ 45 \mathrm{ppm}+$ $\mathrm{GA}_{3} @ 30$ ppm and Factor- B had three levels ofnumber of spray viz. $\mathrm{N}_{0}$-control (no spray), $\mathrm{N}_{1}$ Two spray (vegetative+early flowering stage), $\mathrm{N}_{2}$-three spray (vegetative+early flowering $+80 \%$ flowering stage).

\subsection{Raising of seedling}

Seeds were sown in the seedbed on 5 November 2016. The soil of the experimental plot was treated with savin 50WP at $5 \mathrm{~kg}$ per ha to protect the young plants from the attack of ants and cutworm. The size of the each plot was $1.5 \times 1.2$ $\mathrm{m}$ with 36 plots.

\subsection{Transplanting of seedling}

About 25 days old seedlings were transplanted into the prepared plot on 1 December 2016 with plant to plant spacing $50 \times 30 \mathrm{~cm}$. Fertilizers were applied at 250,330, 250, 110 and 10 ton per ha for urea, TSP, MP, gypsum, and cow dung, respectively according to BARI (2011). Harvesting of fruits was done by hand picking.

\section{Results and Discussion}

\subsection{Days from transplanting to $1^{\text {st }}$ flowering}

No significant variation was observed in terms of initiation of flowering due to imposition of treatments.

\subsection{Number of flowers per plant}

The maximum number of flowers per plant (32.58) was recorded by the application of $\mathrm{G}_{3}$ treatment, whereas the minimum number (30.06) was obtained from $\mathrm{G}_{0}$ treatment (Table 1). Similarly, maximum number of flowers per plant (31.68) was recorded from $\mathrm{N}_{2}$ treatment while the minimum number (30.64) was found from $\mathrm{N}_{0}$ treatment (Table 2). The highest number of flowers per plant (33.33) was recorded from $\mathrm{G}_{3} \mathrm{~N}_{2}$ treatment combination, while the lowest number (29.66) was found from $\mathrm{G}_{0} \mathrm{~N}_{0}$ treatment combination (Table 3). So, more flowers are produced where hormones are applied in combination than control. It was noticed that application of 4-CPA $+\mathrm{GA}_{3}$ enhanced flower production, reduced flower abscission that contributed the maximum number of flower per plant compared to plants treated with others hormone and control. This result is in agreement with the findings of Choudhury et al. (2013) who found that, the highest number of flowers per plant (39.69) were obtained in combined application of $20 \mathrm{ppm} 4-\mathrm{CPA}$ and $20 \mathrm{ppm} \mathrm{GA}_{3}$ in summer tomato plant.

\subsection{Number of fruits per plant}

Number of fruits per plant of sweet pepper showed significant differences due to the effect 
of different plant growth regulators. The maximum number of fruits per plant (12.81) was found from $\mathrm{G}_{3}$ treatment and the minimum number (9.89) was recorded from $\mathrm{G}_{0}$ treatment (Table 1).

Table 1. Main effect of plant growth regulators on flower and fruit setting in sweet pepper

\begin{tabular}{lllllll}
\hline Treatments & $\begin{array}{l}\text { Days from } \\
\text { transplanting } \\
\text { to1 }{ }^{\text {st }} \text { flowering }\end{array}$ & $\begin{array}{l}\text { Number of } \\
\text { flowers } \\
\text { per plant }\end{array}$ & $\begin{array}{l}\text { Number } \\
\text { of fruits } \\
\text { per plant }\end{array}$ & $\begin{array}{l}\text { Number of } \\
\text { marketable } \\
\text { fruits per plant }\end{array}$ & $\begin{array}{l}\text { Fruit } \\
\text { Setting } \\
(\%)\end{array}$ & $\begin{array}{l}\text { Days from } \\
\text { transplanting } \\
\text { to } 1^{\text {st }} \text { harvest }\end{array}$ \\
\hline $\mathrm{G}_{0}$ & 49.16 & $30.06 \mathrm{~d}$ & $9.89 \mathrm{~d}$ & $5.37 \mathrm{~d}$ & $31.45 \mathrm{~d}$ & $121.52 \mathrm{a}$ \\
$\mathrm{G}_{1}$ & 50.25 & $30.65 \mathrm{c}$ & $10.46 \mathrm{c}$ & $5.93 \mathrm{c}$ & $34.09 \mathrm{c}$ & $117.80 \mathrm{~b}$ \\
$\mathrm{G}_{2}$ & 51.38 & $31.51 \mathrm{~b}$ & $11.56 \mathrm{~b}$ & $6.52 \mathrm{~b}$ & $36.65 \mathrm{~b}$ & $113.44 \mathrm{c}$ \\
$\mathrm{G}_{3}$ & 51.98 & $32.58 \mathrm{a}$ & $12.81 \mathrm{a}$ & $7.24 \mathrm{a}$ & $39.09 \mathrm{a}$ & $107.99 \mathrm{~d}$ \\
\hline $\mathrm{CV} \%$ & 11.43 & 7.45 & 8.62 & 5.81 & 4.88 & 5.39 \\
\hline
\end{tabular}

In a column means having similar letter(s) are significantly different per 0.05 level of probability

Table 2. Main effect of number of spray on flower and fruit setting in sweet pepper

\begin{tabular}{lllllll}
\hline Treatments & $\begin{array}{l}\text { Days from } \\
\text { transplanting } \\
\text { to1 }^{\text {st }} \text { flowering }\end{array}$ & $\begin{array}{l}\text { Number of } \\
\text { flowers } \\
\text { per plant }\end{array}$ & $\begin{array}{l}\text { Number } \\
\text { of fruits } \\
\text { per plant }\end{array}$ & $\begin{array}{l}\text { Number of } \\
\text { marketable } \\
\text { fruits per plant }\end{array}$ & $\begin{array}{l}\text { Fruit } \\
\text { Setting } \\
(\%)\end{array}$ & $\begin{array}{l}\text { Days from } \\
\text { transplanting } \\
\text { to } 1^{\text {st }} \text { harvest }\end{array}$ \\
\hline $\mathrm{N}_{0}$ & 49.96 & $30.64 \mathrm{c}$ & $9.90 \mathrm{c}$ & $5.58 \mathrm{c}$ & $32.27 \mathrm{c}$ & $118.39 \mathrm{a}$ \\
$\mathrm{N}_{1}$ & 50.71 & $31.29 \mathrm{~b}$ & $11.33 \mathrm{~b}$ & $6.27 \mathrm{~b}$ & $35.99 \mathrm{~b}$ & $114.76 \mathrm{~b}$ \\
$\mathrm{~N}_{2}$ & 51.41 & $31.68 \mathrm{a}$ & $12.03 \mathrm{a}$ & $6.94 \mathrm{a}$ & $37.86 \mathrm{a}$ & $112.42 \mathrm{c}$ \\
\hline $\mathrm{CV} \%$ & 11.43 & 7.45 & 8.62 & 5.81 & 4.88 & 5.39 \\
\hline
\end{tabular}

In a column means having similar letter(s) are significantly differents per 0.05 level of probability

Table 3. Combined effect of plant growth regulators and number of spray on yield contributing characters and yield of sweet pepper

\begin{tabular}{lllllll}
\hline $\begin{array}{l}\text { Treat } \\
\text { ments }\end{array}$ & $\begin{array}{l}\text { Days from } \\
\text { transplanting } \\
\text { to1 } 1^{\text {st }} \text { flowering }\end{array}$ & $\begin{array}{l}\text { Number } \\
\text { of flowers } \\
\text { per plant }\end{array}$ & $\begin{array}{l}\text { Number } \\
\text { of fruits } \\
\text { per plant }\end{array}$ & $\begin{array}{l}\text { Number of } \\
\text { marketable } \\
\text { fruits per plant }\end{array}$ & $\begin{array}{l}\text { Fruit } \\
\text { Setting } \\
(\%)\end{array}$ & $\begin{array}{l}\text { Days from } \\
\text { transplanting to } \\
1^{\text {st }} \text { harvest }\end{array}$ \\
\hline $\mathrm{G}_{0} \mathrm{~N}_{0}$ & 48.86 & $29.66 \mathrm{~g}$ & $8.92 \mathrm{k}$ & $5.04 \mathrm{j}$ & $30.08 \mathrm{l}$ & $122.28 \mathrm{a}$ \\
$\mathrm{G}_{0} \mathrm{~N}_{1}$ & 49.15 & $29.98 \mathrm{fg}$ & $9.12 \mathrm{j}$ & $5.32 \mathrm{i}$ & $31.72 \mathrm{k}$ & $121.54 \mathrm{~b}$ \\
$\mathrm{G}_{0} \mathrm{~N}_{2}$ & 49.46 & $30.54 \mathrm{def}$ & $10.12 \mathrm{i}$ & $5.75 \mathrm{~h}$ & $33.14 \mathrm{~h}$ & $120.74 \mathrm{c}$ \\
$\mathrm{G}_{1} \mathrm{~N}_{0}$ & 49.33 & $30.15 \mathrm{efg}$ & $9.61 \mathrm{j}$ & $5.37 \mathrm{i}$ & $31.87 \mathrm{j}$ & $119.42 \mathrm{~d}$ \\
$\mathrm{G}_{1} \mathrm{~N}_{1}$ & 50.18 & $30.81 \mathrm{de}$ & $10.54 \mathrm{~g}$ & $5.87 \mathrm{~g}$ & $34.22 \mathrm{f}$ & $117.74 \mathrm{e}$ \\
$\mathrm{G}_{1} \mathrm{~N}_{2}$ & 51.25 & $30.99 \mathrm{~cd}$ & $11.21 \mathrm{e}$ & $6.55 \mathrm{~d}$ & $36.17 \mathrm{e}$ & $116.23 \mathrm{~g}$ \\
$\mathrm{G}_{2} \mathrm{~N}_{0}$ & 50.72 & $31.00 \mathrm{~cd}$ & $10.23 \mathrm{~h}$ & $5.79 \mathrm{gh}$ & $33.00 \mathrm{i}$ & $117.58 \mathrm{f}$ \\
$\mathrm{G}_{2} \mathrm{~N}_{1}$ & 51.36 & $31.68 \mathrm{~b}$ & $11.79 \mathrm{~d}$ & $6.38 \mathrm{e}$ & $37.22 \mathrm{~d}$ & $113.51 \mathrm{i}$ \\
$\mathrm{G}_{2} \mathrm{~N}_{2}$ & 52.07 & $31.85 \mathrm{~b}$ & $12.65 \mathrm{c}$ & $7.39 \mathrm{c}$ & $39.73 \mathrm{c}$ & $109.23 \mathrm{j}$ \\
$\mathrm{G}_{3} \mathrm{~N}_{0}$ & 50.95 & $31.73 \mathrm{~b}$ & $12.65 \mathrm{c}$ & $6.12 \mathrm{f}$ & $34.10 \mathrm{~g}$ & $114.27 \mathrm{~h}$ \\
$\mathrm{G}_{3} \mathrm{~N}_{1}$ & 52.15 & $32.69 \mathrm{a}$ & $13.47 \mathrm{~b}$ & $7.51 \mathrm{~b}$ & $40.79 \mathrm{~b}$ & $106.23 \mathrm{k}$ \\
$\mathrm{G}_{3} \mathrm{~N}_{2}$ & 52.84 & $33.33 \mathrm{a}$ & $14.13 \mathrm{a}$ & $8.10 \mathrm{a}$ & $42.39 \mathrm{a}$ & $103.47 \mathrm{l}$ \\
\hline $\mathrm{CV} \mathrm{CV}$ & 11.43 & 7.45 & 8.62 & 5.81 & 4.88 & 5.39 \\
\hline
\end{tabular}

In a column means having similar letter(s) do not differ significantly at 0.05 level of probability. 
Similarly the maximum number of fruits per plant (12.03) was obtained from $\mathrm{N}_{2}$ treatment, while the minimum number (9.90) was obtained from $\mathrm{N}_{0}$ treatment (Table 2). Combine effect shows that the maximum number of fruits per plant (14.13) was recorded from $\mathrm{G}_{3} \mathrm{~N}_{2}$ treatment, while the minimum number (8.92) was found from $\mathrm{G}_{0} \mathrm{~N}_{0}$ treatment (Table 3). Maximum number of fruit was recorded in plant growth regulators (4-CPA $\left.+\mathrm{GA}_{3}\right)$ treated plants compared to control. This might be occurred due to application of auxin at the time of flowering which resulted lower flower drop and maximum number of fruits per plant.

\subsection{Number of marketable fruits per plant}

Application of plant growth regulators significantly enhanced fruit quality of sweet pepper. The maximum number of marketable fruits per plant (7.24) was found from plants under $\mathrm{G}_{3}$ treatment, while the minimum number (5.37) was obtained from $\mathrm{G}_{0}$ treatment (Table 1). The maximum number of marketable fruits per plant (6.94) was found from $\mathrm{N}_{2}$ treatment, while the minimum number (5.58) from $\mathrm{N}_{0}$ treatment (Table 2). From the results of the present study indicated that different number of spray can affect the fruit quality. The maximum number of marketable fruits per plant (8.10) was recorded from $\mathrm{G}_{3} \mathrm{~N}_{2}$ treatment combination, while the minimum number (5.04) was for $\mathrm{G}_{0} \mathrm{~N}_{0}$ treatment (Table 3). Application of plant growth regulators significantly enhanced fruit.

\subsection{Fruit setting percentage}

The maximum fruit setting (39.09\%) was obtained from $\mathrm{G}_{3}$ treatment, while the minimum fruit setting $(31.45 \%)$ was found from $G_{0}$ treatment (Table 1). The maximum fruit setting (37.86\%) was found from $\mathrm{N}_{2}$ treatment, while the minimum (32.27\%) was recorded from $\mathrm{N}_{0}$ treatment (Table 2). The highest fruit setting $(42.39 \%)$ was observed from $\mathrm{G}_{3} \mathrm{~N}_{2}$ treatment combination, while the lowest $(30.08 \%)$ was found from $\mathrm{G}_{0} \mathrm{~N}_{0}$ treatment combination (Table
3 ). This result is in agreement with the findings of Sasaki et al. (2005) where he obtained that the tomato plants treated with a mixture of 4-CPA and $\mathrm{GA}_{3}$ showed increased fruit set and proportion of normal fruits compared to plants of the same crop treated with 4-CPA alone.

\subsection{Days from transplanting to $1^{\text {st }}$ harvest}

The minimum days from transplanting to $1^{\text {st }}$ harvest (107.99) was found from $\mathrm{G}_{3}$ treatment, while the maximum days (121.52) was recorded from $\mathrm{G}_{0}$ treatment (Table 1). However, minimum days from transplanting to $1^{\text {st }}$ harvest $(112.42)$ was attained from $\mathrm{N}_{2}$ treatment, while the maximum days (118.39) was found from $\mathrm{N}_{0}$ treatment (Table 2). The minimum days from transplanting to $1^{\text {st }}$ harvest $(103.47)$ was recorded from $\mathrm{G}_{3} \mathrm{~N}_{2}$ treatment combination, while the maximum days (122.28) was found from $\mathrm{G}_{0} \mathrm{~N}_{0}$ treatment combination (Table 3 ). Hasanuzzaman et al. (2007) reported that, plant hormones promoted the harvesting of sweet pepper a few days earlier than control.

\subsection{Length and diameter of fruits}

The maximum length $(7.59 \mathrm{~cm})$ and diameter of fruit $(5.33 \mathrm{~cm})$ were found from $\mathrm{G}_{3}$ treatment, where the minimum length $(6.14 \mathrm{~cm})$ and the minimum diameter $(4.25 \mathrm{~cm})$ were found from $\mathrm{G}_{0}$ treatment (Table 4). Plant growth regulators have possibility to increase fruit length and diameter. This result is in agreement with the findings of Hasanuzzaman et al. (2007). The maximum length $(7.39 \mathrm{~cm})$ and diameter of fruit $(5.09 \mathrm{~cm})$ were recorded from $\mathrm{N}_{2}$ treatment, while the minimum length $(6.29 \mathrm{~cm})$ and minimum diameter $(4.42 \mathrm{~cm})$ were found from $\mathrm{N}_{0}$ treatment (Table 5). From the results of the present study indicated that different number of spray can affect the fruit quality. The maximum length of fruit $(8.11 \mathrm{~cm})$ and diameter of fruit $(5.71 \mathrm{~cm})$ were found from $\mathrm{G}_{3} \mathrm{~N}_{2}$ treatment combination, while the minimum length (5.75 $\mathrm{cm})$ and diameter $(4.1 \mathrm{~cm})$ were found from $\mathrm{G}_{0} \mathrm{~N}_{0}$ treatment combination (Table 6). 
Table 4. Main effect of plant growth regulators on yield contributing characters and yield of sweet pepper

\begin{tabular}{lllllll}
\hline Treatment & $\begin{array}{l}\text { Length of } \\
\text { fruit }(\mathrm{cm})\end{array}$ & $\begin{array}{l}\text { Diameter of } \\
\text { fruit }(\mathrm{cm})\end{array}$ & $\begin{array}{l}\text { Pericarp } \\
\text { thickness }(\mathrm{mm})\end{array}$ & $\begin{array}{l}\text { Individual fruit } \\
\text { weight }(\mathrm{g})\end{array}$ & $\begin{array}{l}\text { Yield per } \\
\text { plant }(\mathrm{g})\end{array}$ & $\begin{array}{l}\text { Yield per } \\
\text { hectare (ton) }\end{array}$ \\
\hline $\mathrm{G}_{0}$ & $6.14 \mathrm{~d}$ & $4.25 \mathrm{~d}$ & $5.42 \mathrm{~d}$ & $52.70 \mathrm{~d}$ & $283.56 \mathrm{a}$ & $18.80 \mathrm{~d}$ \\
$\mathrm{G}_{1}$ & $6.71 \mathrm{c}$ & $4.55 \mathrm{c}$ & $5.67 \mathrm{c}$ & $53.73 \mathrm{c}$ & $318.94 \mathrm{c}$ & $21.24 \mathrm{c}$ \\
$\mathrm{G}_{2}$ & $7.13 \mathrm{~b}$ & $4.88 \mathrm{~b}$ & $5.97 \mathrm{~b}$ & $55.38 \mathrm{~b}$ & $361.80 \mathrm{~b}$ & $24.11 \mathrm{~b}$ \\
$\mathrm{G}_{3}$ & $7.59 \mathrm{a}$ & $5.33 \mathrm{a}$ & $6.33 \mathrm{a}$ & $57.38 \mathrm{a}$ & $416.90 \mathrm{a}$ & $27.70 \mathrm{a}$ \\
\hline $\mathrm{CV} \%$ & 6.31 & 8.87 & 9.35 & 10.93 & 9.34 & 9.63 \\
\hline
\end{tabular}

In a column means having similar letter(s) are significantly differents per 0.05 level of probability

Table 5. Main effect of number of spray on yield contributing characters and yield of sweet pepper

\begin{tabular}{lllllll}
\hline Treatment & $\begin{array}{l}\text { Length of } \\
\text { fruit }(\mathrm{cm})\end{array}$ & $\begin{array}{l}\text { Diameter of Pericarp } \\
\text { fruit }(\mathrm{cm})\end{array}$ & $\begin{array}{l}\text { Individual fruit } \\
\text { thickness }(\mathrm{mm})\end{array}$ & $\begin{array}{l}\text { Yield per } \\
\text { weight }(\mathrm{g})\end{array}$ & $\begin{array}{l}\text { Yield per } \\
\text { plant }(\mathrm{g})\end{array}$ & hectare (ton) \\
\hline $\mathrm{N}_{0}$ & $6.29 \mathrm{c}$ & $4.42 \mathrm{c}$ & $5.42 \mathrm{c}$ & $53.40 \mathrm{c}$ & $298.34 \mathrm{c}$ & $19.87 \mathrm{c}$ \\
$\mathrm{N}_{1}$ & $6.99 \mathrm{~b}$ & $4.74 \mathrm{~b}$ & $5.89 \mathrm{~b}$ & $55.08 \mathrm{~b}$ & $347.02 \mathrm{~b}$ & $23.11 \mathrm{~b}$ \\
$\mathrm{~N}_{2}$ & $7.39 \mathrm{a}$ & $5.09 \mathrm{a}$ & $6.16 \mathrm{a}$ & $55.95 \mathrm{a}$ & $390.54 \mathrm{a}$ & $26.00 \mathrm{a}$ \\
\hline $\mathrm{CV} \%$ & 6.31 & 8.87 & 9.35 & 10.93 & 9.34 & 9.63 \\
\hline
\end{tabular}

In a column means having similar letter(s) are significantly different per 0.05 level of probability

Table 6. Interaction of plant growth regulators and number of spray on yieldcontributing characters and yield of sweet pepper

\begin{tabular}{lllllllll}
\hline $\begin{array}{l}\text { Treat } \\
\text { ment }\end{array}$ & $\begin{array}{l}\text { Length } \\
\text { of fruit } \\
(\mathrm{cm})\end{array}$ & $\begin{array}{l}\text { Diameter } \\
\text { of fruit } \\
(\mathrm{cm})\end{array}$ & $\begin{array}{l}\text { Pericarp } \\
\text { thickness } \\
(\mathrm{mm})\end{array}$ & $\begin{array}{l}\text { Individual } \\
\text { fruit } \\
\text { weight }(\mathrm{g})\end{array}$ & $\begin{array}{l}\text { Yield per } \\
\text { plant }(\mathrm{g})\end{array}$ & $\begin{array}{l}\text { Yield per } \\
\text { hectare } \\
(\text { ton })\end{array}$ & $\begin{array}{l}\text { Net } \\
\text { return } \\
(\mathrm{Tk} / \mathrm{ha})\end{array}$ & BCR \\
\hline $\mathrm{G}_{0} \mathrm{~N}_{0}$ & $5.75 \mathrm{~h}$ & $4.10 \mathrm{~g}$ & $5.19 \mathrm{~h}$ & $52.02 \mathrm{k}$ & $262.71 \mathrm{l}$ & $17.50 \mathrm{k}$ & 433045 & 1.49 \\
$\mathrm{G}_{0} \mathrm{~N}_{1}$ & $6.12 \mathrm{~g}$ & $4.19 \mathrm{f}$ & $5.40 \mathrm{~g}$ & $52.66 \mathrm{j}$ & $280.69 \mathrm{k}$ & $18.67 \mathrm{j}$ & 433045 & 1.49 \\
$\mathrm{G}_{0} \mathrm{~N}_{2}$ & $6.54 \mathrm{f}$ & $4.49 \mathrm{e}$ & $5.67 \mathrm{f}$ & $53.44 \mathrm{~h}$ & $307.30 \mathrm{i}$ & $20.43 \mathrm{~h}$ & 433045 & 1.49 \\
$\mathrm{G}_{1} \mathrm{~N}_{0}$ & $6.15 \mathrm{~g}$ & $4.24 \mathrm{f}$ & $5.35 \mathrm{~g}$ & $52.84 \mathrm{i}$ & $283.73 \mathrm{j}$ & $18.90 \mathrm{i}$ & 490585 & 1.53 \\
$\mathrm{G}_{1} \mathrm{~N}_{1}$ & $6.75 \mathrm{e}$ & $4.50 \mathrm{e}$ & $5.67 \mathrm{f}$ & $53.56 \mathrm{~g}$ & $314.38 \mathrm{~g}$ & $20.93 \mathrm{f}$ & 622789 & 1.66 \\
$\mathrm{G}_{1} \mathrm{~N}_{2}$ & $7.23 \mathrm{~d}$ & $4.91 \mathrm{~d}$ & $5.99 \mathrm{de}$ & $54.79 \mathrm{f}$ & $358.70 \mathrm{~d}$ & $23.89 \mathrm{~d}$ & 828423 & 1.87 \\
$\mathrm{G}_{2} \mathrm{~N}_{0}$ & $6.45 \mathrm{f}$ & $4.51 \mathrm{e}$ & $5.56 \mathrm{f}$ & $53.65 \mathrm{~g}$ & $310.63 \mathrm{~h}$ & $20.72 \mathrm{~g}$ & 637450 & 1.69 \\
$\mathrm{G}_{2} \mathrm{~N}_{1}$ & $7.25 \mathrm{~d}$ & $4.88 \mathrm{~d}$ & $6.09 \mathrm{~d}$ & $55.95 \mathrm{~d}$ & $356.89 \mathrm{e}$ & $23.79 \mathrm{~d}$ & 856518 & 1.93 \\
$\mathrm{G}_{2} \mathrm{~N}_{2}$ & $7.69 \mathrm{c}$ & $5.23 \mathrm{c}$ & $6.26 \mathrm{c}$ & $56.54 \mathrm{c}$ & $417.89 \mathrm{c}$ & $27.82 \mathrm{c}$ & 1158085 & 2.25 \\
$\mathrm{G}_{3} \mathrm{~N}_{0}$ & $6.82 \mathrm{e}$ & $4.86 \mathrm{~d}$ & $5.88 \mathrm{e}$ & $54.94 \mathrm{e}$ & $336.30 \mathrm{f}$ & $22.38 \mathrm{e}$ & 225238 & 2.24 \\
$\mathrm{G}_{3} \mathrm{~N}_{1}$ & $7.83 \mathrm{~b}$ & $5.41 \mathrm{~b}$ & $6.41 \mathrm{~b}$ & $58.15 \mathrm{~b}$ & $436.13 \mathrm{~b}$ & $29.05 \mathrm{~b}$ & 1218423 & 2.27 \\
$\mathrm{G}_{3} \mathrm{~N}_{2}$ & $8.11 \mathrm{a}$ & $5.71 \mathrm{a}$ & $6.70 \mathrm{a}$ & $59.05 \mathrm{a}$ & $478.27 \mathrm{a}$ & $31.87 \mathrm{a}$ & 1416558 & 2.46 \\
\hline $\mathrm{CV}_{2}$ & 6.31 & 8.87 & 9.35 & 10.93 & 9.34 & 9.63 & 433045 & 1.49 \\
\hline
\end{tabular}

In a column means having similar letter(s) do not differ significantly at 0.05 level of probability. 


\subsection{Paricarp thickness}

The higher pericarp thickness $(6.33 \mathrm{~mm})$ was recorded from $\mathrm{G}_{3}$ treatment, while the lower thickness $(5.42 \mathrm{~mm})$ was observed from $\mathrm{G}_{0}$ treatment (Table 4). The maximum pericarp thickness $(6.16 \mathrm{~mm})$ was recorded from $\mathrm{N}_{2}$ treatment, while the minimum thickness (5.50 $\mathrm{mm}$ ) was observed from $\mathrm{N}_{0}$ treatment (Table 5). The maximum pericarp thickness $(6.70 \mathrm{~mm})$ was found from $\mathrm{G}_{3} \mathrm{~N}_{2}$ treatment combination, while the minimum thickness $(5.19 \mathrm{~mm})$ was recorded from $\mathrm{G}_{0} \mathrm{~N}_{0}$ treatment combination (Table 6).

\subsection{Individual fruit weight}

The maximum weight of individual fruit (57.38 g) was recorded from $\mathrm{G}_{3}$ treatment, while the minimum weight $(52.70 \mathrm{~g})$ was observed from $\mathrm{G}_{0}$ treatment (Table 4). The maximum weight of individual fruit $(55.95 \mathrm{~g})$ was found from $\mathrm{N}_{2}$ treatment, while the minimum weight $(53.40 \mathrm{~g})$ was recorded from $\mathrm{N}_{0}$ treatment (Table 5). The maximum weight of individual fruit $(59.05 \mathrm{~g})$ was attained from $\mathrm{G}_{3} \mathrm{~N}_{2}$ treatment combination, while the minimum weight $(52.02 \mathrm{~g})$ was found from $\mathrm{G}_{0} \mathrm{~N}_{0}$ treatment combination (Table 6).

\subsection{Fruit yield}

The maximum yield per plant $(416.90 \mathrm{~g})$ and yield per hectare (27.70 ton) were recorded from $\mathrm{G}_{3}$ treatment, while the minimum, yield per plant $(283.56 \mathrm{~g})$ and fruit yield per hectare (18.80 ton) were found from $\mathrm{G}_{0}$ treatment (Table 4). Kannan et al. (2009) reported that application of $\mathrm{GA}_{3}$ had significant effect on growth and yield attributes on peperika hot pepper. The maximum yield per plant (390.54 g) and hectare (26.00 ton) were observed from $\mathrm{N}_{2}$ treatment, while the minimum yield per plant (298.34 g) and yield per hectare (19.87 ton) were recorded from $\mathrm{N}_{0}$ treatment (Table 5). The highest yield per plant $(478.27 \mathrm{~g})$ and yield per hectare (31.87 ton) was attained from $\mathrm{G}_{3} \mathrm{~N}_{2}$ treatment combination, while the lowest yield per plant $(262.71 \mathrm{~g})$ and yield per hectare (17.50 ton) were found from $\mathrm{G}_{0} \mathrm{~N}_{0}$ treatment combination (Table 6). From the results of the study indicated that plant growth regulators can affect the fruit quality. Hasanuzzaman et al. (2007) reported that, due to hormonal treatments significant variation exists in respect of fruit yield. The results revealed that the maximum growth, yield and yield attributes were found with PGRs than control.

\subsection{Net return}

In case of net return, different treatment combination showedvarious levels of net return under the present trial. The highest net return (Tk. 1416558) was obtained from the treatment combination $\mathrm{G}_{3} \mathrm{~N}_{2}$ and the lowest (Tk. 433045) was found from $\mathrm{G}_{0} \mathrm{~N}_{0}$ treatment combination (Table 6)

\subsection{Benefit Cost Ratio}

The highest benefit cost ratio (2.46) was found from the treatment combination of $\mathrm{G}_{3} \mathrm{~N}_{2}$ and the lowest (1.49) was found from the $\mathrm{G}_{0} \mathrm{~N}_{0}$ treatment combination. From the economic point of view, it was apparent that the treatment combination of $\mathrm{G}_{3} \mathrm{~N}_{2}$ was more profitable than others.

\section{Conclusions}

Considering the results of this experiment, it may be concluded that the plant growth regulators (4CPA@ @4 ppm+ GA 3 @ 30 ppm ) and three sprays would give better performance than others. However, the experiment should be carried out with more variables in different AEZs to reconfirm the recommendation.

\section{Acknowledgements}

This study was done under Sher-e Bangla Agricultural University, Dhaka-1207. The authors acknowledge the funding support of the project for National Science and Technology (NST) Fellowship.

\section{References}

Asian Vegetable Research and Development Center. 1989. Tomato and the pepper production in the tropics. Asian Vegetable Research and Development Center, Taiwan. p. 585. 
Bangladesh Agricultural Research Institute. 2011. Krishi Projukti Hatboi, 5th edition, $1^{\text {st }}$ part. December 2011. pp. 484.

Choudhury S., Islam N., Sarkar M.D., Ali M.A. 2013. Growth and yield of summertomato as influenced by plant growth regulators. International Journal of Sustainable Agricultural, 5(1):25-28.

Changli Z., Liusheng K. 2009. The effect of composite method of plant growthregulators, nitrogen fertilizer,and planting density on the yield and quality of hotpepper (Capsicum frutescens). Acta Agriculture Universitatis Jiangxiensis, China. 31: (4) 644 - 649

Erickson A.N., Markhart. 2001. Flower production, fruit set and physiology of bellpepper during elevated temperature and vapor pressure deficit. Journal of the American Society for Horticultural Science, 126(6): 697-702.
Hasanuzzaman S.M., Hossain S.M.M., Ali M.O., Hossain M.A., Hannan A. 2007. Performance of different bell pepper (Capsicum annuum L.) genotypes inresponse to synthetic hormones. International Journal of Sustainable Crop Production, 2:78-84.

Kannan K., Jawaharlal M., Prabhu M. 2009. Auxins especially NAA had positiveeffect on plant growth, early flowering, yield and quality attributes. Agricultural Revolution, 30(3):46-49.

Sasaki H., Yano T. and Yamasaki A. 2005. Reduction of high temperature inhibitionin tomato fruit set by plant growth regulators. Japan agricultural Research Quarterly, 39:135-13.

Vandana P., Verma L.R. 2014. Effect of spray treatment of growth substances at different stages on growth and yield of sweet pepper (Capsicum annum L.) $c v$. Indra under green house. International Journal of Life Sciences Research, 2(4):235-240. 